\title{
Impaired Fasting Glucose alone or in combination with Hypertension, Dyslipidemia, or both AND Major Adverse Cardiovascular Events among patients without previous cardiovascular disease.
}

Nahid Hashemi Madani

endocrine research center, iran niversity $f$ meical science

Faramarz Ismail-Beigi

case western reserve university, scholl of medicine

Hossein Poustchi

digestive diease research center,tehran university of medical science

Mahdi Nalini

digestive disease research center,tehran university of medical science

Sadaf G. Sepanlou

digestive disease research center, tehran university of medical science

Mojtaba Malek

research center for prevention of vascular disease, iran university of medical science

Mohammad Amin Abbasi

endocrine research center, iran university of medical science

Alireza Khajavi

student research center, faculty of paramedical science, shahid beheshti university of medical science

Mohammad Ebrahim Khamseh ( $\nabla$ me.khamseh@gmail.com)

Endocrine Research Center, Institute of Endocrinology and Metabolism, Iran Un https://orcid.org/0000-00034313-8440

Reza Malekzadeh

digestive research center, tehran university of medical science

\section{Research article}

Keywords: Dyslipidemia (DLP), Hypertension (HTN), Impaired fasting glucose (IFG), Major adverse cardiovascular events (MACE), Pre-diabetes (pre-DM).

Posted Date: August 13th, 2019

DOI: https://doi.org/10.21203/rs.2.10473/v2 
License: (1) This work is licensed under a Creative Commons Attribution 4.0 International License. Read Full License 


\section{Abstract}

Background Whether pre-diabetes in the absence of hypertension (HTN) or dyslipidemia (DLP) is a risk factor for occurrence of major adverse cardiovascular events (MACE) is not fully established. We investigated the effect of impaired fasting glucose (IFG) alone and in combination with HTN, DLP or both on subsequent occurrence of MACE as well as individual MACE components. Methods This longitudinal population-based study included 9,831 inhabitants of Northeastern Iran. The participants were free of any cardiovascular disease at baseline and were followed yearly from 2010 to 2017. Cox proportional hazard models were fitted to measure the hazard of IFG alone or in combination with HTN and DLP on occurrence of MACE as the primary endpoint. Results 297 MACE were recorded during 6.2 \pm 0.1 years follow up. IFG alone compared to normal fasting glucose (NFG) was not associated with increased in occurrence of MACE $(\mathrm{HR}, 1.05 ; 95 \% \mathrm{Cl}$, 0.59-1.86; $p, 0.8)$. However, combination of IFG and HTN (HR, 2.75; 95\% Cl, 1.93-3.90; $p, 0.001)$ or HTN + DLP $(\mathrm{HR}, 2.85 ; 95 \% \mathrm{Cl}, 1.79-4.54 ; \mathrm{p}, 0.001)$ significantly increased the hazard of MACE. In contrast, IFG with DLP at baseline did not increase the hazard of MACE compared to normoglycemic- normolipemic individuals $(p, 0.2)$. The results also indicated IFG with HTN, DLP, or HTN+DLP were associated with significant higher HRs for some individual components of MACE. Conclusion IFG, per se, does not appear to increase hazard of MACE. However, IFG with HTN or HTN + DLP conferred a significant hazard for MACE in an incremental manner.

\section{Background}

Pre-diabetes (pre-DM) is often taken as a warning sign. Individuals with pre-DM have a glycemic state that is higher than normal but not high enough to be classified as type 2 diabetes (T2DM). The pre-diabetes state is characterized by impaired fasting glucose (IFG), impaired glucose tolerance (IGT), or HbA1c of $39 \mathrm{mmol} / \mathrm{mol}$ (5.7\%) to $46 \mathrm{mmol} / \mathrm{mol}(6.4 \%)$ [1]. The significance of pre-DM is the associated risk for progression to T2DM which is disproportionately greater at the higher end of the pre-DM range and with the combined presence of IFG and IGT [1]. Pre-DM and T2DM are parts of a continuous spectrum that share in their pathophysiology, and are associated with a common phenotype that includes obesity, hypertension (HTN), and dyslipidemia (DLP) [2]. It is well established that aggregation of the traditional cardiovascular (CV) risk factors such as HTN and DLP in patients with T2DM is associated with the increased risk of cardiovascular disease (CVD) and events $[3,4]$. Furthermore, insulin resistance per se that is present in the vast majority of individuals with T2DM is associated with the increased risk of CVD independent of other CV risk factors $[5,6]$.

In addition to T2DM, pre-DM is also associated with an increased risk of CVD $[7,8]$.Considering that traditional $\mathrm{CV}$ risk factors are frequently present in individuals with pre-diabetes, the question arises that to what extent is the effect of pre-DM on CVD risk mediated by having pre-DM alone or by the associated risk factors [9, 10].Since some studies did not fully adjust for concomitant CV risk factors, their findings should be interpreted with caution. Hence, whether pre-DM or IFG alone in the absence of HTN or DLP or their combination carries increased risk for CVD has remained an unanswered issue.

We conducted a prospective cohort study among a population of Iranian people to investigate whether prediabetes in the absence of HTN or DLP is a risk factor for occurrence of major adverse cardiovascular events as well as its individual components. 


\section{Material And Method}

\section{Study design and population}

We extracted data from the longitudinal Golestan Cohort Study (GCS) that was launched in Northeastern Iran [11]. The primary aim of GCS was to investigate risk factors of esophageal cancer in this region. Participants in this cohort were also followed for CVD outcomes. The participants were men and women aged 40-75 years, without any current or previous history of any known CVD events. In this analysis we examined data from a total number of 11,374 participants that were enrolled from rural and urban areas between April 2010 and March 2012. Participants were followed annually till March 2017. The study protocol was approved by the ethical review committees of the Digestive Disease Research Institute of Tehran University of Medical Sciences, the US National Cancer Institute, and the International Agency for Research on Cancer. Before interview, the written informed consent was obtained from each participant. They were interviewed by a trained general physician who has recorded demographic and medical information through a questionnaire.

Each subject was interviewed by a trained general physician, either in the local language (Turkmen) or in the national formal language (Persian), depending on the participant's preference. Questions were asked about the age, residence area (urban or rural), smoking status, alcohol consumption, history of any heart disease, or any kind of cancer. Height, weight, waist and hip circumference were measured using standard methods, and body mass index (BMI) was calculated. Blood pressure (BP) was measured twice, with a 10-min interval, from both arms in the sitting position using Richter auscultator sphygmomanometers. Blood samples (10 ml) were collected by a trained technician. In the urban area, the samples were immediately processed in the central laboratory at the Golestan Cohort Study Center. In the rural areas, blood samples were kept in refrigerators $(+4 \mathrm{C})$, until they were transferred in cooling boxes to the central laboratory; the maximum duration between blood collection and final processing was $8 \mathrm{~h}$.

Fasting blood glucose (FBS), triglyceride (TG), cholesterol, and high density lipoprotein (HDL) were measured enzymatically in all individuals [11].

\section{Follow up}

Participants were followed up by annual phone calls from their date of recruitment (2010-2012) until the first occurrence of any of MACE components, death from any cause, or end of the study (March 2017). Each cohort member was also instructed at the time of enrollment to contact the team in case of certain conditions such as hospitalization or development of a new major disease. These contacts were registered and subsequently followed by a staff member. In case a death, cancer or any CVD event is reported, the GCS team visited the participant's home and the medical centers in which any major diagnostic or therapeutic procedures were done. The team collects all clinical reports, pathology reports and hospital records. Follow-up assessed at the end of the Golestan study was successful for over $99 \%$ of the study participants. If a death was identified, a general practitioner visited the home of the deceased. Causes of death were ascertained through verbal interview and investigation of medical documents [12]. Using the collected documents, two internists 
independently determined the cause of death with ICD-10 codes. If the two were concordant, a diagnosis was made. Otherwise, the cause of death was determined by a third senior internist.

\section{Definition of IFG, HTN, and DLP}

IFG was diagnosed when a participant did not have DM, but had an FBS of 5.6 to $<7.0 \mathrm{mmol} / \mathrm{L}$, according to the American Diabetes Association criteria (ADA) [1]. Patients with diagnosed T2DM, defined as a self-reported diagnosis, or being treated with glucose lowering drugs, or those with FBS $\geq 7 \mathrm{mmol} / \mathrm{L}$ were excluded from the current analysis. Normal fasting glucose (NFG) was defined as FBS $<5.6 \mathrm{mmol} / \mathrm{L}$. Hypertension was defined as self-reported hypertension and currently taking antihypertensive drugs, or systolic blood pressure (SBP) $\geq 140 \mathrm{~mm} \mathrm{Hg}$ or diastolic blood pressure (DBP) $\geq 90 \mathrm{~mm} \mathrm{Hg}$ based on the JNC-7 criteria [13]. DLP was defined as fasting TG $\geq 1.69 \mathrm{mmol} / \mathrm{L}$, or total cholesterol $\geq 6.20 \mathrm{mmol} / \mathrm{L}$, or $\mathrm{HDL}<1.29 \mathrm{mmol} / \mathrm{L}$ in women and $<1.03$ $\mathrm{mmol} / \mathrm{L}$ in men, or receiving lipid lowering medications.

\section{Statistical Analysis:}

Data are presented as means (SE) and numbers (percentage). In all cases, final analysis was based on presence or absence of a risk factor (or factors) in both the IFG and NGT groups using fully adjusted models. Student t-test and Pearson X2 (or Fisher Exact) tests were used to compare continuous and categorical variables, respectively. Risk analysis was conducted in patients with IFG, and Poisson regression model was used to identify predictors of MACE (fatal MI, fatal stroke, non-fatal MI, and non-fatal stroke). Regarding the time to event for MACE and its components, Kaplan-Meyer method was used to estimate the survival probabilities, and Cox proportional hazard models were fitted to measure the hazard ratios. Statistical analyses were performed with Stata software for Windows version 13.0. Values of $p<0.05$ was considered statistically significant.

\section{Results}

\section{Baseline characteristics}

The entire prospective cohort included 11,374 participants from the Northern provinces of Iran. Among the cohort 1,543 had DM at baseline and were excluded from this analysis. Among the remaining 9,831 participants, 2,410 (24.5\%) had IFG, and 7,421 (75.5\%) had normal fasting glucose (NFG), and they were included in this analysis.

Baseline characteristics of the study population are demonstrated in Table 1. The prevalence of HTN (44.2 vs. $34.7 \%$;, 0.000$)$ and DLP (49.9 vs. 39.7\%; p, 0.000) were significantly higher in IFG population compared to the normoglycemic group. Moreover, compared to NFG group, the IFG group were older and had a significantly higher level of BMI, waist circumference (WC), SBP, DBP, TG, cholesterol, and low density lipoprotein (LDL) $(p=0.000$, all). Participants with IFG were more likely to use a statin medication than did the NFG group (3.7 vs. 
2.7\%; p, 0.007). However, sex difference (47.3 vs. 48.4\%; p, 0.3), smoking status (8.1 vs. 9.2\%; $p, 0.08)$, and HDL level (61.4 vs. 61.2; $p, 0.7)$ were not significantly different between the two groups.

\section{IFG and CVD outcomes}

Over a mean follow up of 6.2 ( \pm 0.1) years, 297 MACE (199 CVD deaths, 43 non-fatal MI, and 55 non-fatal strokes) were reported. CVD death comprised of 108 fatal MI, 74 fatal strokes, and 17 deaths due to heart failure.

Table 2 shows HRs and 95\% Cl of MACE according to glycemic status, HTN, and DLP. The occurrence of MACE was not significantly different between those with IFG but without HTN or DLP compared to NFG participants who also did not have HTN or DLP (fully adjusted HR, 1.05; 95\% Cl, 0.59-1.86; p, 0.8). However, presence of both HTN and DLP with IFG was associated with significantly higher hazard of MACE compared to the reference group (fully adjusted $\mathrm{HR}, 2.85 ; 95 \% \mathrm{Cl}, 1.79-4.54 ; \mathrm{p}, 0.000$ ). The presence of HTN+DLP in normoglycemic participants confers a considerable hazard for MACE (fully adjusted HR, 2.58; $95 \% \mathrm{Cl}, 1.72-$ 3.87; P, 0.000), however, it is somewhat less than hazard of MACE imposed by HTN+ DLP in individuals with IFG. When participants were categorized based on glycemic status with the presence or absence of HTN alone, individuals with IFG plus HTN showed significantly higher hazard of MACE compared to those with NFG and without HTN (fully adjusted HR, 2.75; 95\% Cl, 1.93-3.90; p, 0.000). However, IFG without HTN did not increase the hazard of MACE compared to NFG without HTN (fully adjusted HR, 1.19; 95\% Cl, 0.77-1.84; $p, 0.4$ ). The presence of HTN in normoglycemic participants was associated with significantly higher HR of MACE compared to the reference group (fully adjusted $\mathrm{HR}, 2.57 ; 95 \% \mathrm{Cl}, 1.92-3.43 ; \mathrm{P}, 0.000$ ). HRs for MACE was also evaluated according to glycemic status in the presence or absence of DLP. Compared to the reference group, IFG with DLP did not increase the hazard of MACE (fully adjusted HR, 1.24; 95\% Cl, 0.88-1.77; $p, 0.2$ ).

Kaplan-Meier analysis with log- rank test shows that addition of IFG to HTN, DLP, or both did not decrease MACE free survival (Figure $1 ; p>0.05$ for all comparisons).

We next examined the effect of IFG with HTN, DLP, or both HTN + DLP on the occurrence of individual components of MACE in greater detail. Table 3 shows the effect of IFG in the presence of HTN+DLP on the occurrence of the individual components of MACE. IFG plus both HTN and DLP was associated with a significant increase in the hazard of fatal MI (fully adjusted $\mathrm{HR}, 2.74 ; 95 \% \mathrm{Cl}, 1.20-6.21 ; \mathrm{p}, 0.01$ ). However, IFG in the absence of either HTN or DLP did not increase the hazard of fatal MI (fully adjusted $\mathrm{HR}, 1.20 ; 95 \% \mathrm{Cl}$, 0.47- 3.01; P, 0.6). The combination of IFG with both HTN and DLP did not increase the risk of non-fatal MI (fully adjusted HR, 1.12; $95 \% \mathrm{Cl}, 0.23-5.46 ; \mathrm{p}, 0.9$ ) compared with NFG in the absence of HTN+DLP. Considerably, IFG in the presence of both HTN and DLP conferred a significantly higher hazard (fully adjusted) of both fatal stroke (HR, 5.13; 95\% Cl, 2.08-12.63; p, 0.000) and non-fatal stroke (HR, 2.52; 95\% Cl, 1.06-6.02; p, 0.04), compared to NFG in the absence of HTN+DLP. However, IFG in the absence of either HTN or DLP was not associated with increased hazard of fatal (fully adjusted $\mathrm{HR}, 1.37 ; 95 \% \mathrm{Cl}, 0.43-4.40 ; \mathrm{p}, 0.6$ ) or non-fatal stroke (fully adjusted HR, 0.52; 95\% Cl, 0.12-2.30; p, 0.4).

The results indicated that IFG in the presence of HTN is associated with a significantly higher hazard of fatal MI (fully adjusted HR, 2.66; 95\% Cl, 1.48-24.78; $p, 0.001$ ) but not non-fatal MI (fully adjusted $\mathrm{HR}, 1.57 ; 95 \% \mathrm{Cl}$, 0.56- 4.46; p, 0.4) compared to NFG in the absence of HTN (Table 4). IFG plus HTN was associated with the 
higher hazard of both fatal (fully adjusted HR, 3.72; 95\% Cl, 1.84-7.55; p, 0.000) and non-fatal (fully adjusted $\mathrm{HR}, 2.34 ; 95 \% \mathrm{Cl}, 1.18-4.62 ; \mathrm{p}, 0.01)$ stroke. However, IFG in the absence of HTN did not increase the hazard of any of the above cardiovascular events (Table 4).

IFG in the presence of DLP, compared to NFG in the absence of DLP, was associated with an increased risk of fatal stroke both in unadjusted model (HR, 2.17; 95\% Cl, 1.22-3.88; $p, 0.009)$ and in adjusted models number $1(\mathrm{HR}, 2.40 ; 95 \% \mathrm{Cl}, 1.33-4.31 ; \mathrm{p}, 0.003)$ and number 2 (HR, 2.47; 95\% Cl, 1.34-4.53; $\mathrm{p}, 0.004)$. However, IFG in the presence of DLP did not increase the risk of fatal MI (fully adjusted HR, 1.50; $95 \% \mathrm{Cl}, 0.84-2.70 ; \mathrm{p}, 0.2$ ), nonfatal MI (fully adjusted $\mathrm{HR}, 0.72 ; 95 \% \mathrm{Cl}, 0.21-2.52 ; \mathrm{p}, 0.6$ ), and non-fatal stroke (fully adjusted $\mathrm{HR}, 1.31 ; 95 \% \mathrm{Cl}$, 0.66-2.58; $p, 0.4)$. Likewise, IFG in the absence of DLP did not increase the hazard of any of the above cardiovascular events (Table 5).

\section{Discussion}

The major findings of the present study on composite CVD outcomes (MACE) in a group of individuals with IFG are that IFG, per se, in the absence of HTN and DLP is not associated with an increase in the hazard for MACE. In contrast, IFG in the presence of HTN or HTN+DLP is associated with an incremental and significant increase in the hazard of MACE. Further analyses regarding the impact of IFG in the presence of HTN, DLP, or HTN+DLP on the individual components of MACE demonstrated that IFG in the presence of HTN or HTN+DLP incrementally increases the hazard of fatal MI as well as fatal and non-fatal stroke. Additionally, the combination of IFG and DLP is associated with an increase in the risk of fatal stroke.

Whether pre-DM, per se, is an independent risk factor for CVD has been a matter of debate. In the current study, IFG in the absence of other CV risk factors did not increase the risk of adverse cardiovascular events. Likewise, other longitudinal population-based studies, also did not find any association between pre-DM alone and CVD risk $[14,15]$. However, other studies have indicated that dysglycemia within pre-diabetic glucose range was associated with an increase in the risk of CVD $[6,16,17]$. Most studies have suggested that impaired glucose tolerance (IGT) is a stronger predictor than IFG for CVD events $[18,19]$. However, Barr et al. showed IFG but not IGT is an independent predictor for CVD mortality [20]. Furthermore, a recently published study demonstrated the incidence of MACE in patients with established coronary artery disease (CAD) and pre-DM defined as either of IFG or IGT does not differ from those in patients with normal glycemic status [21]. However, a previous study conducted to assess the risk of death according to the different diagnostic glucose categories showed "fasting blood glucose alone is not sufficient to predict mortality related to hyperglycemia" They found in any category of fasting glucose, an increase in the 2-h post-load glucose is associated with a higher risk of mortality [22]. Likewise, results from a recent review suggested that IGT and HbA1C correlate more with CVD risk than IFG [23]. These inconsistencies might be due to differences in analytical methods, different definitions for pre- diabetes, and ethnic origins of the participants included in the studies.

It is generally agreed that individuals with pre-diabetes or T2DM have higher than normal CV risk factors. This is probably due to the fact that T2DM and pre-DM share common underlying pathophysiologic disturbances such as insulin resistance [2]. In our study, as expected, participants with IFG had significantly higher BMI, WC, SBP, DBP, TG, total cholesterol, and LDL compared to normoglycemic subjects. Moreover, they were more likely 
to have HTN and DLP than those with NFG. Similar results are frequently reported in previous studies investigating the presence of $\mathrm{CV}$ risk factors in individuals with pre-DM $[9,10,24]$.

Aggregation of CV risk factors in individuals with pre-DM is known to place them at higher risk for CV events [10]. In the current study we found that IFG when associated with HTN significantly increased the risk of composite end-point of MACE. This result is in line with the results of some previous studies in which the coexistence of pre-DM and HTN, but not pre-DM without HTN, significantly increased the risk of CVD events $[14,15]$. Another study showed that pre-DM defined as IFG or IGT even in the presence of prehypertension, defined as SBP between120 to 139 or DBP between 80 to $89 \mathrm{~mm} \mathrm{Hg}$, significantly increases the risk for CVD events [25]. HTN is a well-known and powerful risk factor for almost all different types of CVD events [26]. Similarly, the current analysis indicated HTN alone in normoglycemic participants is associated with higher HRs for both MACE and all individual components of MACE. The reason why coexistence of pre-DM and HTN, but not pre-DM without HTN, leads to higher CVD events might reflect the fact that both of them induce endothelial dysfunction and inflammation through elevation of inflammatory factors such as tumor necrosis factor a (TNF a) and intercellular adhesion molecule-1 (ICMA-1) [27]. Hence, co-occurrence of HTN, or even preHTN, with pre-DM results in higher levels of inflammatory factors and more severe endothelial damage conferring additive risk for subsequent occurrence of composite end-point of MACE.

DLP, characterized by elevated TG, low concentration of HDL, and an increased number of small dense LDL, plays a critical role in acceleration of atherosclerosis and is reported as an important independent risk factor for CVD [28]. Several studies have demonstrated that individuals with pre-diabetes have deranged lipid profiles compared to normoglycemic subjects $[9,22]$. The frequent association of DLP with pre-DM, which may reflect the underlying insulin resistance, has led to frequent treatment of DLP in the pre-diabetes [29, 30]. Despite of the importance of screening and treatment of DLP in individuals with pre-diabetes, we have not found studies that combined pre-DM and DLP together in the analysis for predicting CVD risk. Nevertheless, when we compared the impact of IFG plus DLP with that of NFG without DLP, we found that coexistence of DLP and IFG did not confer any additional risk for development of the composite end-point of MACE. One possible explanation for this finding is that individuals with IFG were more likely to use statin compared to normoglycemic individuals. Nowadays, statin therapy is widely recommended for both primary and secondary prevention of CV disease in a wide range of individuals, even in those without hyperlipidemia [31, 32]. Statins exert their beneficial effects not only through lowering of LDL, but also by improving endothelial function [33], reducing vascular inflammation [32,34], and exerting antithrombotic actions [35].

With regard to the addition of both HTN+DLP to IFG, the present analyses showed an incremental increase in the occurrence of composite end-point of MACE. Although addition of DLP to IFG did not increase the hazard of MACE, co-existence of DLP+HTN increased the hazard for MACE even more than addition of HTN alone to IFG. Our findings support the results of previous studies showing that the risk of CV events associated with coexistence of HTN and DLP, labeled as "lipitation", is greater than the sum of the CV risks for HTN and DLP alone [36]. The interaction between these two risk factors occurs at the vascular endothelial level and results in increased oxidative stress and endothelial dysfunction, disproportionate vascular contractility, elevation of BP in dyslipidemic patients, further elevation of BP in hypertensive patients, and accelerates the development and progression of atherosclerotic lesions [37]. A similar finding has been reported in a large recent study conducted among patients with 5 years' duration of T2DM. The results of this study showed that patients with 
T2DM who had no CV risk factors had little or no excess risk of CVD events or death compared with general population with no diabetes [38]. The findings highlight the significance of control of CV risk factors both in patients with pre-DM and T2DM.

Considering the effect of IFG combined with CV risk factors on the individual components of MACE, we found that IFG did not increase the hazard of any of the individual outcomes in the absence of other CV risk factors. Addition of DLP, HTN, or HTN+DLP to IFG was associated with an incremental increase in the hazard of fatal stroke. In addition, IFG combined with HTN alone or HTN+DLP significantly increased the hazard of non-fatal stroke. However, IFG combined with DLP was not associated with an increase in the hazard of non-fatal stroke. The reason why the risk of non-fatal stroke did not increase in such an incremental manner might be due to the beneficial effect of antihypertensive therapy that is shown to prevent the occurrence of non-fatal stroke but not death [39]. Moreover, the results showed IFG in the presence of HTN or HTN + DLP is associated with an increase in the hazard of fatal MI but not non-fatal MI. However, IFG combined with DLP did not increase the hazard of fatal and non-fatal MI. These inconclusive findings could be due to the small number of events in each group. In addition, further use of statin might protect participants with IFG + DLP from some individual CV events.

This study has several strengths. It is the first study that investigated the impact of IFG combined with HTN, DLP, or HTN + DLP, on the occurrence of MACE. We included an adequately large number of participants without any known CVD at baseline and the study had $99 \%$ follow-up of participants. In addition, we adjusted for multiple confounding factors including most of the traditional CVD risk factors. Finally, the occurrence of individual CV outcomes, including fatal $\mathrm{Ml}$, fatal stroke, non-fatal $\mathrm{MI}$, and non-fatal stroke, was assessed. Weaknesses include the fact that, due to non-availability of $2 \mathrm{~h}$ post-prandial blood glucose, the effect of only IFG as a component of pre-diabetes could be examined. Although evidence shows that IFG is useful for screening of pre-diabetes in the general population, use of IGT and HbA1C might be stronger predictors of cardiovascular events $[19,40]$. Finally, considering the study design, the findings might be subjected to unmeasured confounding factors. In addition, risk factors were only measured at baseline. Therefore, the impact of the changes could not be evaluated.

\section{Conclusions}

In summary, although IFG, per se, did not increase the hazard of MACE, the association of IFG with HTN or HTN+ DLP conferred a significant hazard of MACE in an incremental manner. These findings support the idea that emphasis should be placed on identification and treatment of multiple $\mathrm{CV}$ risk factors in people with prediabetes.

\section{Abbreviations}

Pre-DM: pre-diabetes; T2DM: type 2 diabetes; IFG: impaired fasting glucose; IGT: impaired glucose tolerance; HTN: hypertension; DLP: dyslipidemia; CV: cardiovascular; CVD: cardiovascular disease; MACE: major adverse cardiovascular events; GCS: Golestan Cohort Study; BMI: body mass index; BP: blood pressure; FBS: fasting blood glucose; TG: triglyceride; HDL: high density lipoprotein; ADA: American Diabetes Association criteria; 
DBP: diastolic blood pressure; SBP: systolic blood pressure; NFG: normal fasting glucose; WC: waist circumference; LDL: low density lipoprotein.

\section{Declarations}

\section{Ethics approval and consent to participate}

The study protocol was approved by the ethical review committees of the Digestive Disease Research Institute of Tehran University of Medical Sciences, the US National Cancer Institute, and the International Agency for Research on Cancer. Before interview, the written informed consent was obtained from each participant.

\section{Consent for publication}

Not applicable.

\section{Availability of data and material}

The datasets used and/or analyzed during the current study are available from the corresponding author on reasonable request.

\section{Competing interests}

The authors declare that they have no competing interests.

\section{Funding}

No funding was received for this work.

\section{Authors' contributions}

M.E.Kh and M.M contributed to study concept and design. N.H.M and M.A.A drafted the manuscript. H.P and M.N, acquired data. R.M and S.G.S contributed to the analysis and interpretation of data. F.I.B critically revised the manuscript. A.Kh contributed to statistical analysis. M.E.Kh is the guarantor of this work and, as such, has full access to all the data in the study and takes responsibility for the integrity of the data and the accuracy of the data analysis.

\section{Acknowledgements}

Not applicable.

\section{References}

1. American Diabetes A. 2. Classification and Diagnosis of Diabetes. Diabetes care. 2017; 40 (Suppl 1): S11S24. 2. Ferrannini E, Gastaldelli A, lozzo P. Pathophysiology of prediabetes. The Medical clinics of North America. 2011; 95(2):327-339. 3. Tatsumi Y, Ohkubo T. Hypertension with diabetes mellitus: significance from an epidemiological perspective for Japanese. Hypertension research: official journal of the Japanese Society of Hypertension. 2017;40 (9):795-806. 4. Warraich HJ, Rana JS. Dyslipidemia in diabetes mellitus and 
cardiovascular disease. Cardiovascular Endocrinology. 2017; 6 (1):27-32. 5. Hashemi Madani N, Ismail-Beigi F, Khamseh ME, Malek M, Ebrahimi Valojerdi A. Predictive and explanatory factors of cardiovascular disease in people with adequately controlled type 2 diabetes. European journal of preventive cardiology. 2017; 24(11):1181-1189. 6. Chien KL, Hsu HC, Su TC, Chen MF, Lee YT, Hu FB. Fasting and postchallenge hyperglycemia and risk of cardiovascular disease in Chinese: The Chin-Shan Community Cardiovascular Cohort study. American heart journal. 2008; 156(5):996-1002. 7. Huang Y, Cai X, Mai W, Li M, Hu Y. Association between prediabetes and risk of cardiovascular disease and all-cause mortality: systematic review and metaanalysis. Bmj. 2016; 355: i5953. 8. Ford ES, Zhao G, Li C. Pre-diabetes and the risk for cardiovascular disease: a systematic review of the evidence. Journal of the American College of Cardiology. 2010; 55(13):1310-7. 9. Kansal S, Kamble TK. Lipid Profile in Prediabetes. J Assoc Physicians India. 2016; 64 (3):18-21. 10. Haffner SM SM, Hazuda HP, Mitchell BD, Patterson JK. Cardiovascular risk factors in confirmed prediabetic individuals. Does the clock for coronary heart disease start ticking before the onset of clinical diabetes? JAMA. 1990; 263(21):2893-2898. 11. Pourshams A, Khademi H, Malekshah AF, Islami F, Nouraei M, Sadjadi AR, et al. Cohort Profile: The Golestan Cohort Study-a prospective study of oesophageal cancer in northern Iran. International journal of epidemiology. 2010; 39(1):52-59. 12. Khademi H, Etemadi A, Kamangar F, Nouraie M, Shakeri R, Abaie B, et al. Verbal autopsy: reliability and validity estimates for causes of death in the Golestan Cohort Study in Iran. PloS one. 2010; 5(6): e11183. 13. James PA, Oparil S, Carter BL, Cushman WC, DennisonHimmelfarb C, Handler J, Lackland DT, LeFevre ML, MacKenzie TD, Ogedegbe O, Smith SC Jr, Svetkey LP, Taler SJ, Townsend RR, Wright JT Jr, Narva AS, Ortiz E. 2014 evidence-based guideline for the management of high blood pressure in adults: report from the panel members appointed to the Eighth Joint National Committee (JNC 8). JAMA. 2014; 311(5):507-520. 14. Liu HH, Cao YX, Li S, Guo YL, Zhu CG, Wu NQ, Gao Y, Dong QT, Zhao $X$, Zhang Y, Sun D, Li JJ. Impacts of Prediabetes Mellitus Alone or Plus Hypertension on the Coronary Severity and Cardiovascular Outcomes. Hypertension. 2018; 71(6):1039-1046. 15. Qiu M, Shen W, Song X, Ju L, Tong W, Wang $\mathrm{H}$, Zheng S, Jin Y, Wu Y, Wang W, Tian J. Effects of prediabetes mellitus alone or plus hypertension on subsequent occurrence of cardiovascular disease and diabetes mellitus: longitudinal study. Hypertension. 2015; 65(3):525-30. 16. Laukkanen JA, Makikallio TH, Ronkainen K, Karppi J, Kurl S. Impaired fasting plasma glucose and type 2 diabetes are related to the risk of out-of-hospital sudden cardiac death and all-cause mortality. Diabetes care. 2013; 36(5):1166-71. 17. Selvin E, Lazo M, Chen Y, Shen L, Rubin J, McEvoy JW, Hoogeveen RC, Sharrett AR, Ballantyne CM, Coresh J. Diabetes mellitus, prediabetes, and incidence of subclinical myocardial damage. Circulation. 2014; 130(16):1374-82. 18. Tominaga M, Eguchi H, Manaka H, Igarashi K, Kato T, Sekikawa A. Impaired glucose tolerance is a risk factor for cardiovascular disease, but not impaired fasting glucose. The Funagata Diabetes Study. Diabetes care. 1999; 22(6):920-4. 19. DECODE Study Group the EDEG. Glucose tolerance and cardiovascular mortality: comparison of fasting and 2-hour diagnostic criteria. Arch Intern Med. 2001; 3(161):397-405. 20. Barr EL1, Zimmet PZ, Welborn TA, Jolley D, Magliano DJ, Dunstan DW, Cameron AJ, Dwyer T, Taylor HR, Tonkin AM, Wong TY, McNeil J, Shaw JE. Risk of cardiovascular and all-cause mortality in individuals with diabetes mellitus, impaired fasting glucose, and impaired glucose tolerance: The Australian Diabetes, Obesity, and Lifestyle Study (AusDiab). Circulation. 2007; 116(2):151-7. 21. Kiviniemi AM, Lepojärvi ES, Tulppo MP, Piira OP, Kentta TV, Perkiämäki JS, et al. Prediabetes and Risk for Cardiac Death Among Patients With Coronary Artery Disease: The ARTEMIS Study. Diabetes Care. 2019; 42:1319-25. ä 22. The DECODE study group. Glucose tolerance and mortality: comparison of WHO and American Diabetes Association diagnostic criteria. The DECODE study group. European Diabetes Epidemiology Group. Diabetes Epidemiology: Collaborative analysis Of Diagnostic criteria in Europe. Lancet. 
1999;354: 617-21. 23. Huang D, Refaat M, Mohammedi K, Jayyousi A, Al Suwaidi J, Abi Khalil C. Macrovascular Complications in Patients with Diabetes and Prediabetes. BioMed research international. 2017; 2017:7839101. 24. Vandana Balgi LH, E Sahna, Shinto K Thomas. Pattern of Lipid Profile Abnormality in Subjects with Prediabetes. International Journal of Scientific Study. 2017; 4(11):150-3. 25. Zhang Y1, Lee ET, Devereux RB, Yeh J, Best LG, Fabsitz RR, Howard BV. Prehypertension, diabetes, and cardiovascular disease risk in a population-based sample: The Strong Heart Study. Hypertension. 2006; 47(3):410-4. 26. Lawes CM1, Rodgers A, Bennett DA, Parag V, Suh I, Ueshima H, MacMahon S. Blood pressure and cardiovascular disease in the Asia Pacific region. Journal of hypertension. 2003; 21(4):707-16. 27. Huang Z, Chen C, Li S, Kong F, Shan P, Huang W. Serum Markers of Endothelial Dysfunction and Inflammation Increase in Hypertension with Prediabetes Mellitus. Genet Test Mol Biomarkers. 2016; 20(6):322-7. 28. Murad MH1, Hazem A, Coto-Yglesias F, Dzyubak S, Gupta S, Bancos I, Lane MA, Erwin PJ, Berglund L, Elraiyah T, Montori VM. The association of hypertriglyceridemia with cardiovascular events and pancreatitis: a systematic review and meta-analysis. BMC endocrine disorders. 2012; 12:2. 29. ML. Metabolic Syndrome: Insulin Resistance and Prediabetes. FP Essent. 2015; 435: 11-6. 30. Gagliardino JJ, Elgart JF, Bourgeois M, Etchegoyen G, Fantuzzi G, Ré M, Ricart JP, García S, Giampieri C, González L, Suárez-Crivaro F, Kronsbein P, Angelini JM, Martínez C, Martínez J, Ricart A, Spinedi E. Diabetes primary prevention program: New insights from data analysis of recruitment period. Diabetes Metab Res and Rev. 2018; 34(1). 31. Chou R, Dana T, Blazina I, Daeges M, Jeanne TL. Statins for Prevention of Cardiovascular Disease in Adults: Evidence Report and Systematic Review for the US Preventive Services Task Force. JAMA. 2016; 316(19):2008-24. 32. Ridker PM. Rosuvastatin to Prevent Vascular Events in Men and Women with Elevated C-Reactive Protein. NEJM. 2008; 359:2195-207. 33. Dupuis J TJ, Cernacek P, Théroux P. Cholesterol reduction rapidly improves endothelial function after acute coronary syndromes. The RECIFE (reduction of cholesterol in ischemia and function of the endothelium) trial. Circulation. 1999; 25(99):3227-33. 34. Leffer DJ. Statins as potent antiinflammatory drugs. Circulation. 2002; 106(16):2041-2. 35. Lacoste L LJ, Hung J, Letchacovski G, Solymoss CB, Waters D. Hyperlipidemia and coronary disease. Correction of the increased thrombogenic potential with cholesterol reduction. Circulation. 1995; 11(92):3172-7. 36. Dalal JJ, Padmanabhan TN, Jain P, Patil S, Vasnawala H, Gulati A. LIPITENSION: Interplay between dyslipidemia and hypertension. Indian J of Endocrinol Metab. 2012; 16 (2):240-5. 37. Hurtubise J, McLellan K, Durr K, Onasanya $\mathrm{O}$, Nwabuko D, Ndisang JF. The Different Facets of Dyslipidemia and Hypertension in Atherosclerosis. Curr Atheroscler Rep. 2016; 18(12):82. 38. Rawshani A, Rawshani A, Franzén S, Sattar N, Eliasson B, Svensson AM, Zethelius B, Miftaraj M, McGuire DK, Rosengren A, Gudbjörnsdottir S. Risk Factors, Mortality, and Cardiovascular Outcomes in Patients with Type 2 Diabetes. N Engl J Med. 2018; 379(7):633-44. 39. Gueyffier F, Bulpitt C, Boissel JP, Schron E, Ekbom T, Fagard R, Casiglia E, Kerlikowske K, Coope J. Antihypertensive drugs in very old people: a subgroup meta-analysis of randomized controlled trials. Lancet. 1999; 353(9155):793-6. 40. Warren B, Pankow JS, Matsushita K, Punjabi NM, Daya NR, Grams M, Woodward M, Selvin E. Comparative prognostic performance of definitions of prediabetes: a prospective cohort analysis of the Atherosclerosis Risk in Communities (ARIC) study. The Lancet Diabetes \& Endocrinology. 2017; 5(1):34-42.

\section{Tables}

Table 1 Baseline characteristics of the participants according to the glycemic status. 


\begin{tabular}{lcccc}
\hline \multicolumn{1}{c}{ Variables } & Total (N=9831) & NFG (N=7421) & IFG (N=2410) & p-value* \\
\hline Age(yr.) & $56.0(0.1)$ & $55.8(0.1)$ & $56.7(0.2)$ & 0.000 \\
Sex (\%M) & $48.1 \%$ & $48.4 \%$ & $47.3 \%$ & 0.3 \\
Smoking (\%) & & & & 0.08 \\
Past & $8.1 \%$ & $7.8 \%$ & $8.8 \%$ & \\
Current & $8.9 \%$ & $9.2 \%$ & $8.1 \%$ & \\
Never & $83.0 \%$ & $83.0 \%$ & $83.1 \%$ & \\
Alcohol (\%) & $2.7 \%$ & $2.7 \%$ & $2.6 \%$ & 0.6 \\
Statin (\%) & $2.9 \%$ & $2.7 \%$ & $3.7 \%$ & 0.007 \\
BMI(kg/m $)$ & $26.8(0.1)$ & $26.5(0.1)$ & $27.6(0.1)$ & 0.000 \\
WC(cm) & $93.4(0.1)$ & $92.6(0.2)$ & $96.5(0.4)$ & 0.000 \\
W/H & $0.43(0.001)$ & $0.43(0.001)$ & $0.44(0.001)$ & 0.000 \\
SBP(mmHg) & $124.9(0.2)$ & $123.9(0.2)$ & $128.0(0.4)$ & 0.000 \\
DBP(mmHg) & $77.2(0.1)$ & $76.8(0.1)$ & $78.4(0.3)$ & 0.000 \\
FBS (mmol/L) & $5.15(0.1)$ & $4.87(0.1)$ & $6.00(0.1)$ & 0.000 \\
TG (mmol/L) & $1.49(0.9)$ & $1.43(1.0)$ & $1.64(1.9)$ & 0.000 \\
Chol (mmol/L) & $5.22(0.5)$ & $5.18(0.6)$ & $5.35(0.9)$ & 0.000 \\
LDL (mmol/L) & $3.04(0.6)$ & $3.01(0.7)$ & $3.13(1.0)$ & 0.000 \\
HDL(mmol/L) & $1.59(0.3)$ & $1.58(0.3)$ & $1.59(0.8)$ & 0.7 \\
DLP \% & $4147(42.2 \%)$ & $2945(39.7 \%)$ & $1202(49.9 \%)$ & 0.000 \\
HTN \% & $3642(37.1 \%)$ & $2578(34.7 \%)$ & $1064(44.2 \%)$ & 0.000 \\
\hline
\end{tabular}

Data are presented as mean (Standard error), number (percentage).

* Comparing NFG and IFG groups

BMI; body mass index, WC; waist circumference, W/H; waist to hip ratio, SBP; systolic blood pressure, DBP; diastolic blood pressure, FBS; fasting blood glucose, TG; triglyceride, Chol; cholesterol, LDL; low density lipoprotein, HDL; high density lipoprotein, DLP; dyslipidemia, HTN; hypertension.

Table 2 Cox regression models predicting MACE according to glycemic status, HTN, and DLP. 


\begin{tabular}{cccc}
\hline & \multicolumn{3}{c}{ HR (95\% CI) for incident MACE } \\
\hline Category & Unadjusted model & Adjusted model 1 & Adjusted model 2* \\
NFG/-HTN/-DLP & 1.0 & 1.0 & 1.0 \\
NFG/+HTN/+DLP & $2.81(1.92-4.11)$ & $2.51(1.70-3.69)$ & $2.58(1.72-3.87)$ \\
& $\mathrm{P}=0.000$ & $\mathrm{P}=0.000$ & $\mathrm{P}=0.000$ \\
IFG/-HTN/-DLP & $1.13(0.64-2.02)$ & $1.06(0.59-1.88)$ & $1.05(0.59-1.86)$ \\
& $\mathrm{P}=0.6$ & $\mathrm{P}=0.8$ & $\mathrm{P}=0.8$ \\
IFG/+HTN/+DLP & $3.24(2.10-5.01)$ & $2.77(1.78-4.32)$ & $2.85(1.79-4.54)$ \\
& $\mathrm{P}=0.000$ & $\mathrm{P}=0.000$ & $\mathrm{P}=0.000$ \\
Category & Unadjusted model & Adjusted model 1 & Adjusted model $2^{* *}$ \\
NFG/-HTN & 1.0 & 1.0 & 1.0 \\
NFG/+ HTN & $3.09(2.34-4.09)$ & $2.47(1.86-3.28)$ & $2.57(1.92-3.43)$ \\
& $\mathrm{P}=0.000$ & $\mathrm{P}=0.000$ & $\mathrm{P}=0.000$ \\
IFG/-HTN & $1.22(0.80-1.89)$ & $1.18(0.77-1.82)$ & $1.19(0.77-1.84)$ \\
& $\mathrm{P}=0.3$ & $\mathrm{P}=0.4$ & $\mathrm{P}=0.4$ \\
IFG/+HTN & $3.40(2.43-4.76)$ & $2.67(1.90-3.76)$ & $2.75(1.93-3.90)$ \\
& $\mathrm{P}=0.000$ & $\mathrm{P}=0.000$ & $\mathrm{P}=0.000$ \\
Category & Unadjusted model & Adjusted model 1 & Adjusted model $2^{* * *}$ \\
NFG/-DLP & 1.0 & 1.0 & 1.0 \\
NFG/ + DLP & $0.99(0.75-1.30)$ & $1.12(0.84-1.48)$ & $1.06(0.79-1.41)$ \\
& $\mathrm{P}=0.9$ & $\mathrm{P}=0.4$ & $\mathrm{P}=0.6$ \\
IFG/-DLP & $1.18(0.83-1.67)$ & $1.09(0.77-1.55)$ & $1.05(0.74-1.50)$ \\
& $\mathrm{P}=0.35$ & $\mathrm{P}=0.6$ & $\mathrm{P}=0.7$ \\
IFG/+DLP & $1.34(0.96-1.88)$ & $1.43(1.01-2.01)$ & $1.24(0.88-1.77)$ \\
& $\mathrm{P}=0.08$ & $\mathrm{P}=0.03$ & $\mathrm{P}=0.2$ \\
\hline & & &
\end{tabular}

Model 1: adjusted for age + sex, Model 2*: adjusted for age + sex + BMI + smoking (Never as reference). Model 2**: adjusted for age + sex + BMI + DLP + smoking (Never as reference). Model 2***: adjusted for age + sex + BMI + HTN + smoking (Never as reference). NFG; normal fasting glucose, IFG; impaired fasting glucose, HTN; hypertension, DLP; dyslipidemia.

Table 3 Cox regression models predicting individual components of MACE according to glycemic status, HTN, DLP. 


\begin{tabular}{|c|c|c|c|}
\hline & \multicolumn{3}{|c|}{ HR (95\% CI) for incident fatal MI } \\
\hline Category & Unadjusted model & Adjusted model 1 & Adjusted model 2* \\
\hline NFG/-HTN/-DLP & 1.0 & 1.0 & 1.0 \\
\hline \multirow[t]{2}{*}{ NFG/+HTN/+DLP } & $2.83(1.50-5.36)$ & $2.44(1.28-4.67)$ & $2.87(1.44-5.69)$ \\
\hline & $\mathrm{P}=0.00$ & $\mathrm{P}=0.007$ & $\mathrm{P}=0.003$ \\
\hline \multirow[t]{2}{*}{ IFG/-HTN/-DLP } & $1.25(0.50-3.15)$ & $1.17(0.47-2.93)$ & $1.20(0.47-3.01)$ \\
\hline & $\mathrm{P}=0.6$ & $\mathrm{P}=0.7$ & $\mathrm{P}=0.6$ \\
\hline \multirow[t]{3}{*}{ IFG/+HTN/+DLP } & $2.78(1.29-5.98)$ & $2.31(1.06-5.04)$ & $2.74(1.20-6.21)$ \\
\hline & $\mathrm{P}=0.009$ & $\mathrm{P}=0.04$ & $\mathrm{P}=0.01$ \\
\hline & \multicolumn{3}{|c|}{ HR (95\% CI) for incident non-fatal MI } \\
\hline Category & Unadjusted model & Adjusted model 1 & Adjusted model $2^{* *}$ \\
\hline NFG/-HTN/-DLP & 1.0 & 1.0 & 1.0 \\
\hline \multirow[t]{2}{*}{ NFG/+HTN/+DLP } & $3.94(1.68-9.22)$ & $4.17(1.75-9.91)$ & $3.80(1.51-9.60)$ \\
\hline & $\mathrm{P}=0.002$ & $\mathrm{P}=0.001$ & $\mathrm{P}=0.005$ \\
\hline \multirow[t]{2}{*}{ IFG/-HTN/-DLP } & $0.91(0.20-4.20)$ & $0.87(0.19-4.04)$ & $0.85(0.18-3.97)$ \\
\hline & $\mathrm{P}=0.9$ & $\mathrm{P}=0.8$ & $\mathrm{P}=0.8$ \\
\hline \multirow[t]{3}{*}{ IFG/+HTN/+DLP } & $1.18(0.26-5.48)$ & $1.25(0.27-5.88)$ & $1.12(0.23-5.46)$ \\
\hline & $\mathrm{P}=0.8$ & $\mathrm{P}=0.7$ & $\mathrm{P}=0.9$ \\
\hline & \multicolumn{3}{|c|}{ HR (95\% CI) for incident fatal stroke } \\
\hline Category & Unadjusted model & Adjusted model 1 & Adjusted model $2 * * *$ \\
\hline NFG/-HTN/-DLP & 1.0 & 1.0 & 1.0 \\
\hline \multirow{2}{*}{$\mathrm{NFG/+HTN/+DLP}$} & $2.84(1.18-6.86)$ & 2.45 ( $1.01-5.97)$ & $2.42(0.96-6.12)$ \\
\hline & $\mathrm{P}=0.01$ & $\mathrm{P}=0.05$ & $\mathrm{P}=0.06$ \\
\hline \multirow[t]{2}{*}{ IFG/-HTN/-DLP } & $1.60(0.50-5.11)$ & $1.41(0.44-4.52)$ & $1.37(0.43-4.40)$ \\
\hline & $\mathrm{P}=0.4$ & $\mathrm{P}=0.6$ & $\mathrm{P}=0.6$ \\
\hline \multirow[t]{3}{*}{ IFG/+HTN/+DLP } & $6.43(2.78-14.88)$ & $5.20(2.21-12.24)$ & $5.13(2.08-12.63)$ \\
\hline & $\mathrm{P}=0.000$ & $\mathrm{P}=0.000$ & $\mathrm{P}=0.000$ \\
\hline & \multicolumn{3}{|c|}{ HR (95\% CI) for incident non-fatal stroke } \\
\hline Category & Unadjusted model & Adjusted model 1 & Adjusted model $2^{* * *}$ \\
\hline NFG/-HTN/-DLP & 1.0 & 1.0 & 1.0 \\
\hline \multirow[t]{2}{*}{ NFG/+HTN/+DLP } & $1.54(0.65-3.68)$ & $1.24(0.51-3.00)$ & $1.10(0.44-2.74)$ \\
\hline & $\mathrm{P}=0.3$ & $P=0.6$ & $P=0.8$ \\
\hline \multirow[t]{2}{*}{ IFG/-HTN/-DLP } & 0.58 ( 0.13- 2.57) & $0.55(0.12-2.42)$ & $0.52(0.12-2.30)$ \\
\hline & $\mathrm{P}=0.5$ & $\mathrm{P}=0.4$ & $\mathrm{P}=0.4$ \\
\hline \multirow[t]{2}{*}{ IFG/+HTN/+DLP } & 3.74 ( $1.66-8.41)$ & $2.86(1.24-6.56)$ & $2.52(1.06-6.02)$ \\
\hline & $\mathrm{P}=0.001$ & $\mathrm{P}=0.01$ & $\mathrm{P}=0.04$ \\
\hline
\end{tabular}

Model 1: adjusted for age + sex, Model 2*: adjusted for age + sex + BMI + smoking (Never as reference). Model 2**: adjusted for age + sex + BMI + DLP + smoking (Never as reference). Model 2***: adjusted for age + sex + BMI + HTN + smoking (Never as reference). NFG; normal fasting glucose, IFG; impaired fasting glucose, HTN; hypertension, DLP; dyslipidemia.

Table 4 Cox regression models predicting individual components of MACE according to glycemic status and HTN. 


\begin{tabular}{|c|c|c|c|}
\hline & \multicolumn{3}{|c|}{ HR (95\% CI) for incident fatal MI } \\
\hline Category & Unadjusted model & Adjusted model 1 & Adjusted model 2 \\
\hline NFG/-HTN & 1.0 & 1.0 & 1.0 \\
\hline \multirow[t]{2}{*}{ NFG/+HTN } & $3.02(1.91-4.80)$ & $2.37(1.48-3.79)$ & $2.56(1.59-4.13)$ \\
\hline & $\mathrm{P}=0.000$ & $\mathrm{P}=0.00$ & $\mathrm{P}=0.000$ \\
\hline \multirow[t]{2}{*}{ IFG/-HTN } & $1.41(0.73-2.77)$ & $1.37(0.70-2.67)$ & $1.41(0.72-2.76)$ \\
\hline & $\mathrm{P}=0.3$ & $\mathrm{P}=0.4$ & $\mathrm{P}=0.3$ \\
\hline \multirow[t]{3}{*}{ IFG/+HTN } & $3.16(1.79-5.57)$ & $2.46(1.39-4.38)$ & $2.66(1.48-4.78)$ \\
\hline & $\mathrm{P}=0.000$ & $\mathrm{P}=0.002$ & $\mathrm{P}=0.001$ \\
\hline & \multicolumn{3}{|c|}{ HR (95\% CI) for incident non-fatal MI } \\
\hline Category & Unadjusted model & Adjusted model 1 & Adjusted mc \\
\hline NFG/-HTN & 1.0 & 10 & 1.0 \\
\hline \multirow[t]{2}{*}{ NFG/+HTN } & 1.36- 5.19) & 2.66 & $2.59(1.2$ \\
\hline & $\mathrm{P}=$ & & $\mathrm{P}=$ \\
\hline \multirow[t]{2}{*}{ IFG/-HTN } & $0.72(0$ & 0.71( & $0.70(0.20-2.44)$ \\
\hline & & & \\
\hline \multirow[t]{3}{*}{ IFG/+HTN } & 1.60( & $1.63(0$. & $1.57(0.56-4.46)$ \\
\hline & 0.4 & $\mathrm{P}=0.3$ & $\mathrm{P}=0.4$ \\
\hline & \multicolumn{3}{|c|}{ HR (95\% CI) for incident fatal stroke } \\
\hline Cate & isted model & Adjusted model 1 & Adjusted \\
\hline NFG/-HTN & & & \\
\hline \multirow[t]{2}{*}{ NFG/+HTN } & 3.74 ( $2.04-6.87)$ & $2.73(1.4$ & $2.83(1.52-5.27)$ \\
\hline & $\mathrm{P}=0.000$ & & $\mathrm{P}=0$ \\
\hline \multirow[t]{2}{*}{ IFG/-HTN } & $2.22(1.01-4.90)$ & $2.12(0.96-4.68)$ & $2.10(0.95-4.64)$ \\
\hline & & & \\
\hline \multirow[t]{3}{*}{ IFG/+HTN } & $5.06(2$ & $3.63(1.8$ & $3.72(1.84-7.55)$ \\
\hline & $\mathrm{P}=0.000$ & $\mathrm{P}=0.000$ & $\mathrm{P}=0$ \\
\hline & \multicolumn{3}{|c|}{ HR (95\% CI) for incident non-fatal stroke } \\
\hline Cate & Unadjusted model & Adjusted model 1 & Adjusted mo \\
\hline NFG/-HTN & 1.0 & 1.0 & 1.0 \\
\hline \multirow[t]{2}{*}{ NFG/+HTN } & $2.49(1.43-4.36)$ & $1.83(1.04-3.22)$ & $1.87(1.05-3.33)$ \\
\hline & $\mathrm{P}=0.001$ & & $P=0.03$ \\
\hline \multirow[t]{2}{*}{ IFG/-HTN } & $0.65(0.23-1.90)$ & $0.64(0.22-1.84)$ & $0.63(0.22-1.83)$ \\
\hline & $\mathrm{P}=0.4$ & $\mathrm{P}=0.4$ & $\mathrm{P}=0.4$ \\
\hline \multirow[t]{2}{*}{ IFG/+HTN } & $3.22(1.67-6.21)$ & $2.29(1.18-4.46)$ & $2.34(1.18-4.62)$ \\
\hline & $\mathrm{P}=0.000$ & $\mathrm{P}=0.02$ & $\mathrm{P}=0.01$ \\
\hline
\end{tabular}

1odel 1: adjusted for age + sex, Model 2: adjusted for age + sex + BMI + DLP + smoking (Never as reference). MACE; major adverse iovascular events, NFG; normal fasting glucose, IFG; impaired fasting glucose, HTN; hypertension, DLP; dyslipidemia.

Table 5 Cox regression models predicting individual components of MACE according to glycemic status and DLP. 


\begin{tabular}{|c|c|c|c|}
\hline & \multicolumn{3}{|c|}{ HR (95\% CI) for incident fatal MI } \\
\hline Category & Unadjusted model & Adjusted model 1 & Adjusted model 2 \\
\hline NFG/- DLP & 1.0 & 1.0 & 1.0 \\
\hline \multirow[t]{2}{*}{ NFG/+DLP } & $1.03(0.65-1.6$ & 1.19 ( 0.75- 1 & $1.22(0.76-1.96)$ \\
\hline & $P=0.9$ & $\mathrm{P}=($ & $\mathrm{P}=0.4$ \\
\hline \multirow[t]{2}{*}{ IFG/- DLP } & $1.29(0.73-2.29)$ & $1.20(0.6$ & $1.20(0.68-2.13)$ \\
\hline & $\mathrm{P}=0.4$ & $\mathrm{P}=0.5$ & $\mathrm{P}=0.5$ \\
\hline \multirow[t]{3}{*}{ IFG/+ DLP } & $1.32(0.75-2.34)$ & $1.45(0.82-2.57)$ & $1.50(0.84-2.70)$ \\
\hline & $\mathrm{P}=0.3$ & $\mathrm{P}=0.2$ & $\mathrm{P}=0.2$ \\
\hline & \multicolumn{3}{|c|}{ HR (95\% CI) for incident non-fatal MI } \\
\hline Categ & usted model & Adjusted model 1 & Adjusted $\mathrm{r}$ \\
\hline NFG/- DLP & 1.0 & & 1 \\
\hline \multirow[t]{2}{*}{ NFG/+DLP } & 1.81 ( 0.93- 3.53) & $2.01(1$ & $1.91(0 . \subseteq$ \\
\hline & $\mathrm{P}=0.08$ & $\mathrm{P}=$ & $\mathrm{P}=$ \\
\hline \multirow[t]{2}{*}{ IFG/- DLP } & $1.15(0.42-3.14)$ & $1.12(0.4$ & $1.11(0.41-3.04)$ \\
\hline & $\mathrm{P}=0.8$ & $\mathrm{P}=0.8$ & $\mathrm{P}=0.8$ \\
\hline \multirow{3}{*}{ IFG/+ DLP } & $21-2.42)$ & $0.77(0.2$ & $0.72(0.21-2.52)$ \\
\hline & $\mathrm{P}=0.6$ & $\mathrm{P}=0.7$ & $\mathrm{P}=0.6$ \\
\hline & \multicolumn{3}{|c|}{ HR (95\% CI) for incident fatal stroke } \\
\hline Cate & Unadjusted model & Adjusted m & Adjusted $n$ \\
\hline NFG/- DLP & 1.0 & & \\
\hline \multirow[t]{2}{*}{ NFG/+DLP } & $0.79(0.43-1.45)$ & $0.95(0.52-1.75)$ & $0.99(0.53-1.84)$ \\
\hline & $\mathrm{P}=0.5$ & & \\
\hline \multirow{2}{*}{ IFG/- DLP } & $1.07(0.51-2.24)$ & $0.97(0.46-2.04)$ & $0.98(0.46-2.06)$ \\
\hline & $\mathrm{P}=0.9$ & & $\mathrm{P}=$ \\
\hline \multirow[t]{3}{*}{ IFG/+ DLP } & $2.17(1.22-3.88)$ & $2.40(1.33-4.31)$ & $2.47(1.34-4.53)$ \\
\hline & $\mathrm{P}=0.009$ & $\mathrm{P}=0.003$ & $\mathrm{P}=0.004$ \\
\hline & \multicolumn{3}{|c|}{ HR (95\% CI) for incident non-fatal stroke } \\
\hline Cates & Unadjusted model & Adjusted model 1 & Adjusted mo \\
\hline NFG/- DLP & 1.0 & 1.0 & 1.0 \\
\hline \multirow[t]{2}{*}{ NFG/+DLP } & $0.72(0.40-1.30)$ & $0.78(0.43-1.42)$ & $0.77(0.42-1.42)$ \\
\hline & $\mathrm{P}=0.2$ & & $\mathrm{P}=0.4$ \\
\hline \multirow[t]{2}{*}{ IFG/- DLP } & $0.76(0.34-1.72)$ & $0.71(0.31-1.59)$ & $0.69(0.31-1.56)$ \\
\hline & $\mathrm{P}=0.5$ & $\mathrm{P}=0.4$ & $\mathrm{P}=0.4$ \\
\hline \multirow[t]{2}{*}{ IFG/+ DLP } & $1.33(0.69-2.56)$ & $1.34(0.69-2.60)$ & $1.31(0.66-2.58)$ \\
\hline & $\mathrm{P}=0.4$ & $\mathrm{P}=0.4$ & $\mathrm{P}=0.4$ \\
\hline
\end{tabular}

Model 1: adjusted for age + sex, Model 2: adjusted for age + sex + BMI + HTN + smoking (Never as reference). MACE; major adverse iovascular events, NFG; normal fasting glucose, IFG; impaired fasting glucose; HTN; hypertension, DLP; dyslipidemia.

\section{Figures}



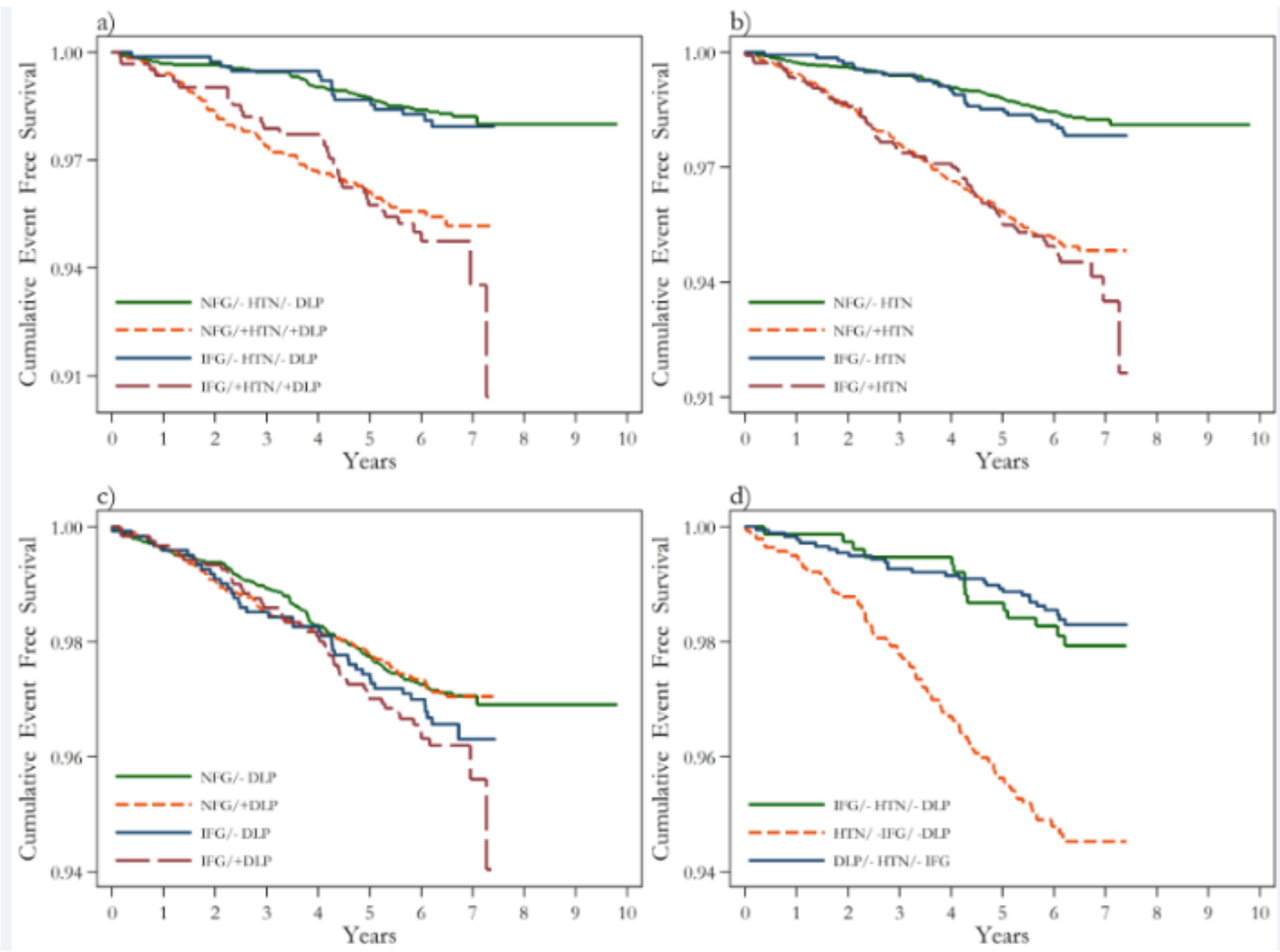

\section{Figure 1}

The cumulative event- free survival analysis for occurrence of MACE according to (a) glycemic status, HTN, and DLP, (b) glycemic status and HTN, (c) glycemic status and DLP, (d) IFG, HTN, and DLP alone. MACE; major adverse cardiovascular events, NFG; normal fasting glucose, IFG; impaired fasting glucose, HTN; hypertension, DLP; dyslipidemia. 